\title{
EL DIARIO EN LA FORMACIÓN EN PRESERVICIO: HIS TORIA DE UN ENCUENTRO EN EL INEF DE GALICIA
}

TOJA REBOREDO, BELÉN
UNTVERSIDAD DE LA CORUNA

CARREIRO DA COSTA, FRANCISCO

UNIVRSIDAD TECNICA DELISBOA
(FACULTAD DE LA MOTRICIDAD HUMANA)

\section{Introducción}

En el INEF de Galicia, al igual que en otros INEFS desde el curso 94/95 se está impartiendo un nuevo plan de estudios, lo cual ha supuesto la aparición de nuevas asignaturas, diferente estructura, distintas exigencias para los alumnos. Con este motivo, durante el bienio 96 98 , se llevó a cabo un programa de tutorización de alumnos en Practicum, que originó una investigación que culminó en una tesis doctoral (Toja, 2001). En ella, se estudió el pensamiento y experiencias de los alumnos que cursan el Practicum, proponiendo la Investigación-AC ción como instrumento de formación. (Toja y otros, 1998 y Toja, 2001).

El objetivo de este artículo no es mostrar las posibilidades del diario en los programas de formación, esto en su momento fue uno de los objetos de estudio de la tesis anteriormente referida, pero esta tarea cubrió cientos de hojas imposible de reducir al espacio de un artículo. Lo que aquí presentamos fue el encuentro en tre las alumnas y dicho instrumento con la intención de que otros profesionales que lo quieran aplicar tengan referencias previas.

Esta experiencia se realizó en el bienio 96/98 quedando resumidas en dos artículos del año 1999 (Toja y otros 1999a y 1999b). Conlleva dos cursos académicos porque el Practicum en el INEF de Galicia se en cuentra estructurado en 2 años, el primero para conocimiento del ámbito y preparación del proyecto y el segundo para la intervención propiamente.

Aunque la mayoría de las referencias que se presentan en esta comunicación pertenecen a los datos de la primera experiencia, que dio lugar a la tesis doctoral durante estos años hemos comprobado que muchos de los resultados se repiten con otros alumnos y con ello se refuerzan las conclusiones iniciales.

Este programa de tutorización intenta utilizar las posibilidades organizativas y estructurales que ofrece la investigación-acción en favor de una dinámica que para nosotros es necesaria en todo proceso de formación "la reflexión en la acción"(Shön, 1983,1987). Es por esto que, para el programa hemos manejado conceptos, elementos, estrategias y técnicas esenciales a ella.
GONZÁLEZ VALETRO, MIGUEL ANGEL
UNIVERSIDAD DE LA CORUNA

Las principales características del programa de formación son:

1. Postura ecléctica respecto a las concepciones en la formación de profesores.

2. Reflexión sobre, en y para la acción.

3. Autogestión de la formación por el grupo y desde sus necesidades.

4. Autoevaluación y análisis de la propia práctica a partir del visionado de la misma (autoscopia).

5. Utilización del diario como instrumento de autorreflexión y de reflexión grupal.

6. Tutorización compartida (seria implicación de los tutores y supervisores).

\section{Formación simultánea inicial y permanente.}

8. Dotar al alumno en prácticas de una Sólida Formación. En un primer momento los investigadoresformadores constituimos un grupo, por lo que se puede hablar de una investigación colaborativa. A continuación aunque existía un plan inicial, todos los miembros del grupo reedificamos un nuevo plan que, debido a la espiral introspectiva, característica del método de investigación-acción, sufrió modificaciones con las que surgieron otros subplanes.

El grupo definitivamente quedó constituido (Toja 2001: Toja y otros 1999a y 1999b) por sujetos con distinto rol (4 alumnos del INEF, 2 profesores supervisores de la Universidad y 2 profesores tutores de centros públicos de enseñanza secundaria), manteniendo una dinámica participativa, democrática y dialogante. La reflexión se tomó como principal objetivo, convirtiéndose en principio y fin del proceso formativo.

A su vez, instrumentos como el diario, que aquí vamos a estudiar detenidamente sirvieron como guía del proceso, facilitaron la reflexión, permitieron la recogida de información e incluso mediaron entre las dimensiones investigación y formación.

Los principales objetivos que perseguíamos al iniciar la investigación-acción fueron los siguientes

- Conocer y describir el pensamiento de las alumnas (motivaciones, creencias, conceptos, expectativas, 
Retos. Nuevas tendencias en

Educación Física, Deporte y Recreación

2002, $n^{\circ} 3, p p .25-30$
Federación Española de Asociaciones de Docentes de Educación Física (FEADEF)

ISSN edición impresa: $1579-1726$ experiencias) antes de empezar la realización del Practicum durante su desarrolloy al finalizarlo.

- Elaborar y orientar un programa de tutorización del Practicum en el área de la Educación Física dentro del marco del paradigma Sociocrítico.

- Describir la evolución de dicho programa a lo largo de los dos cursos académicos que duró.

- Analizar las repercusiones de programa citado en el desarrollo del conocimiento profesional durante el proceso y al finalizar éste.

- Evaluar las posibilidades que algunos instrumentos como el diario y el vídeo tienen en el proceso de reflexión.

- Valorar la influencia que este programa de formación colaborativo ejerce sobre las tradicionales relaciones producidas en la tríada tutor universidad- supervisor del centro de prácticas-alumno/os en prácticas.

\section{El diario como instrumento de formación e in- vestigación}

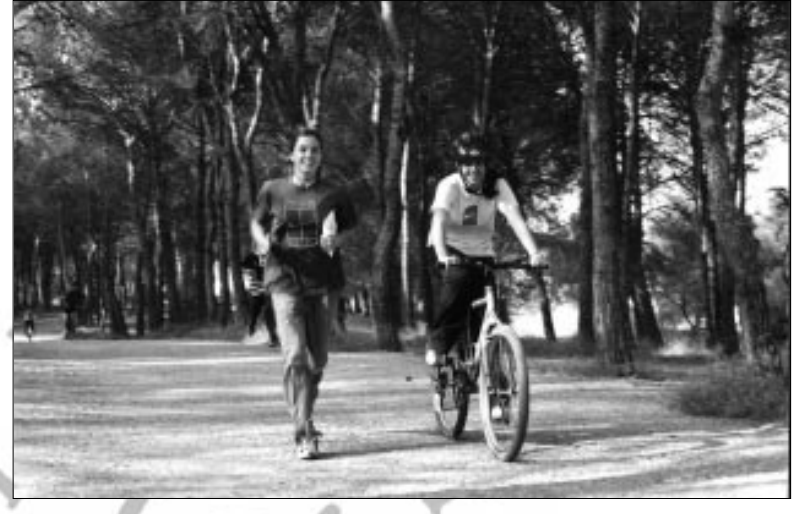

experiencias, preocupaciones, problemas, durante la realización del Practicum De esta misma opinión son autores como Del Villar, 1994: García Ruso, 1992; Yinger y Clark, 1981, 1988; y Zabalza, 1991, que comparten la utilidad del diario como un instrumento adecuado para el análisis del pensamiento y conocimiento del profesor, tanto en formación inicial como permanente.

Finalmente, nos interesaba utilizar el diario porque sus características generales respondían perfectamente a nuestros intereses de formación- investigación.

Características del diario:

- Es un documento permanente en el tiempo.

- Precisa de tiempo para la elaboración y para la reflexión.

- Exige un compromiso personal.

- Favorece el aprendizaje a través de la práctica, la conciencia, el autoconocimiento y la confianza en sí mismo.

- Por el hecho de escribir el conocimiento se hace más consciente, lo cual conlleva un aprendizaje mucho más allá de la "simple" vivencia.

- El escribir proporciona un proceso base para el desarrollo profesional.

Desde el momento inicial en que ideamos este proyecto comprendimos que el diario desempe ñaría necesariamente un papel imprescindible.

Por una parte, conocíamos los buenos resultados que la utilización de esta técnica había cosechado en las experiencias de investigación-acción llevadas a cabo en distintos contextos; por otra nos apoyábamos en las últimas tendencias en formación del profesorado, en las que se propone éste como instrumento favorecedor del proceso de aprendizaje reflexivo.

A su vez, como técnica de recogida de datos favorecía la consecución de uno de nuestros principales objetivos: conocer e pensamiento de los alumnos, sus

\section{El diario en nuestro programa de investigación-formación}

El diario fue presentado al grupo de investigación-acción en la segunda reunión posterior a la constitución del grupo. Inicialmente pretendíamos que todos los miembros elaborásemos un diario a lo largo de la experiencia, aunque posteriormente sólo lo realizaron las alumnas en formación.

Para adecuar las demandas a las posibilidades de ejecución, solicitamos que el diario recogiese tan sólo información acerca de los siguientes momentos:

- Cada una de las reuniones realizadas durante los dos cursos

- Los períodos de observación en el centro de prácticas (Practicum I)

- Todos los días de prácticas en el centro de prácticas (Practicum II)

En la primera presentación ninguno de los miembros del grupo manifestó una actitud negativa hacia su realización, aunque se mostraron ciertas dudas 
respecto a cómo elaborarlo, formato, estructura. Propusimos los siguientes criterios:

- Se trataba de un documento único y personal lo cual por definición lo convertía en válido y útil para atender tanto a los objetivos de formación como a los de investigación.

- Era importante diferenciar aquello que era narra ción de lo que era interpretación o reflexión. Para seguir este criterio se les propusieron diferentes posibilidades: escribir en dos colores, utilizar comillas..., aunque la forma de expresarse era suficiente para diferenciar.

- Se destacaba la importancia de dialogar con el diario, "hablar en voz alta" sobre lo que sucedía en las reuniones, los problemas del Practicum, sus opiniones, explicaciones a su modo de actuar, etc. Se pued observar que los estudiantes hablan más personalmente a través del diario que cara a cara, como puede ocurrir en una entrevista.

- Era asimismo trascendente escribir lo más próximo posible en el tiempo al momento de haber realizado la reunión o haber vivido la experiencia práctica y en la medida de lo posible, adoptar un ritmo periódico de trabajo, intentado evitar las lagunas o que el factor memoria influyera en lo escrito.

- Cuando iniciamos en el Practicum II, se les solicitó que intentasen separar en los diarios de prácticas los aspectos organizativos, los aspectos relacionales con los alumnos, las opiniones sobre su actuación etc. Sin embargo, no se les dio una guía o formato.

etc. Sin embargo, no se les dio una guia o formato. que solamente se haría público al resto del grupo por deseo o interés personal de algún miembro.

- Manifiestamente, perseguíamos que a través del diá logo establecido con ellos mismos gracias a los diarios, llevaran a cabo una auténtica autoevaluación del proceso formativo.

"El diario se convierte, para los futuros profesores, en un elemento facilitador del proceso reflexivo integrador de la teoría y la práctica y estimulador de la implicación personal." (Rodríguez López, 1995: 200)

Presentados estos criterios tan abiertos, que respondían a nuestros objetivos e intereses de investigación y de formación, dimos prácticamente por zanjadas las discusiones sobre su elaboración.

Para facilitar la organización de las alumnas, propusimos la elaboración de dos diarios:

(1) DIARIO DE REUNIONES: Recogió sus vivencias y opiniones respecto a cada una de las reuniones realizadas durante el Practicum I y II. También incluyó las referencias que ellas quisieron hacer respecto a las observaciones realizadas durante el Practicum I.

Ha sido utilizado con una doble funcionalidad: favorecer el proceso formativo/ reflexivo de nuestras alumnas y servir de instrumento de recogida de datos para la investigación.

Una de las razones por las que consideramos oportuna su elaboración fue porque pensamos que inicialmente a las alumnas les resultaría más fácil reflexionar so-

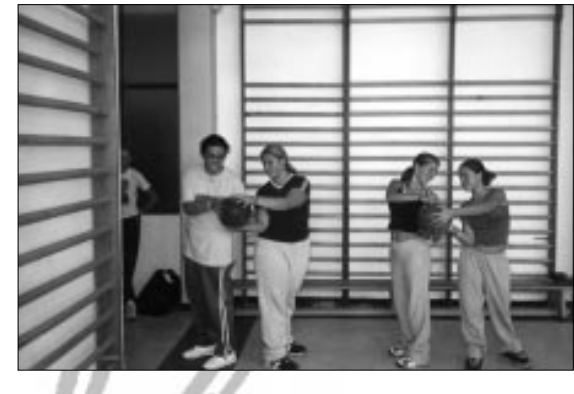

bre lo dicho en las reuniones, e incluso "cuestionar afir maciones", ante su diario que frente a todo el grupo. A su vez, creímos que si el grupo iba funcionando bien, esta modalidad de diario iría siendo progresivamente menos necesaria porque la relación más abierta facilitaría un diálogo fluido y sincero; sin embargo, esto no iba a desvirtuar su utilización, ya que en ese momento jugaría una función de memoria de lo sucedido, más que de interlocutor anónimo.

Atendiendo a su utilidad hemos definido este diario las reuniones, como instrumento para la acción. Su intención era, fundamentalmente, mejorar la futura práctica, la cual entendemos que debe emanar de la reflexión y es fruto, a su vez, de un aprendizaje y hábito previos.

(2) DIARIO DE PRÁCTICAS: En él encontramos todas las reflexiones de las alumnas acerca de cada uno de los días que impartieron docencia en el centro de prácticas.

Asume las funciones de los diarios de reuniones, que estimulaban especialmente una reflexión para la acción añadiendo además una función de análisis en la acción y sobre la acción.

Esta no es una herramienta muy novedosa, pues se utiliza incluso en paradigmas de formación de docentes de carácter tecnicista. Nosotros hemos intentado concienciar a nuestras alumnas de su importancia, de la necesidad de establecer un continuo diálogo persona con el instrumento $y$ de no convertirlo en un simple do

En un principio temimos sobrecargar a las alumnas $y$, en algún momento, hacerlas caer en el desánimo y provocar el abandono. Por el contrario, ocurrió algo similar a lo que hace años les sucedió a Yinger y Clark (1985) cuando iniciaron sus trabajos con diarios. Éstos habían supuesto que sería difícil que los profesores estuviesen dispuestos a realizar el gran esfuerzo que suponía recoger las ideas que les pasaban por la cabeza cuando programaban y escribirlo en sus diarios, por lo cual estaban dispuestos a animarlos mucho y prestarles gran apoyo. Pero los profesores se sintieron cómodos elaborándolos y el diario pronto dejó de ser un instrumento de investigación para otros y pasó a convertirse en un instrumento útil para ellos mismos. De igual modo en un instrú trume (aunque con ciertos miedos) se situaron ante él con ilusión y dedicación. Enseguida pudimos comprobar, primero por sus comentarios y más tarde por la lectura de los propios diarios e incluso de las entrevistas, que éste se había convertido en algo familiar, casi indispensable 
para la reflexión sobre su práctica y muy valorado por ellas. Nuestra experiencia con las alumnas nos permite corroborar las consideraciones de Zabalza (1991), al afirmar que una vez que los profesores se meten dentro de la dinámica del diario le encuentran, normalmente, mucho sentido y una gran utilidad.

El análisis e interpretación de la información de los diarios se presentó desde un principio como una tarea complicada debido a su amplitud. Necesitábamos ordenarla, darle sentido, trasformar y codificar toda esa información (que nos hablaba del pensamiento de nuestras alumnas y de la repercusión del programa), de tal modo que nos permitiese explicar científicamente los resultados de nuestra investigación.

La relevancia dada a estos documentos ha sido tanta que el análisis e interpretación del resto de instrumentos han estado subordinados a las decisiones tomadas respecto al diario: las dimensiones y la mayor parte de las categorías surgieron del análisis de los diarios y, a posteriori, intentamos utilizarlas también, y en la medida de lo posible, para el análisis de los otros documentos, pues entendimos que esto favorecía la triangulación de resultados. Asimismo, tampoco comenzamos a analizar los otros documentos hasta que finalizamos el análisis de los diarios.

\section{El diario analizado por sus protagonistas}

Como ya hemos comentado con anterioridad, el Diario, en concreto aquel realizado durante los periodos de prácticas. no es una herramienta muy novedosa, pues se utiliza incluso en paradigmas de formación de docentes de carácter tecnicista. Nosotros hemos intentado concienciar a las alumnas de su importancia, de la necesidad de establecer un continuo diálogo personal con el instrumento y de no convertirlo en un simple documento descriptivo a modo de memoria de lo sucedido.

Una vez realizado su estudio y análisis detallado, podemos afirmar que las alumnas comprendieron nuestras demandas y en todos los casos se estableció un diálogo fluido con el instrumento.

"El diario me ayuda muchísimo para ver las cosas que me habían quedado en el tintero en las reuniones. Al llegar a casa, te ayudan un poco a reflexionar y tomar una postura, porque a la hora de escribir tenías que tomar una postura,(...)El diario de reuniones yo diría que es fundamental. La labor que cumplió en las reuniones, lo cumplió en las prácticas mucho más, porque necesitabas pararte a pensar, ¿qué hiciste? ¿cómo lo hiciste? ¿qué debías haber cambiado?'(Entrevista a la alumna B. 18 2-98)" (Toja, 2001, p.379)
A pesar de ello, algunos documentos son mucho más detallados que otros, son más descriptivos, ofrecen mayor riqueza para su análisis. Sin embargo, esto era predecible dada la libertad que les dimos para su elaboración. Este hecho confirma que cada diario responde a una forma personal de expresarse, a las propias experiencias vividas $y$, por supuesto, al tipo y nivel de pensamiento.

Tras la triangulación se confirma que las alumnas, igual que viene sucediendo en todas las investigaciones en las que se está utilizando esta técnica (Fraile. 1995; García Ruso, 1992; González Sanmamed, 1994; Marcelo, 1994: Medina, 1995; Ramos, 1999; Romero, 1995: Zabalza, 1991) han valorado positivamente su uso, afirmando que les ha facilitado sus reflexiones tanto de la propia intervención, como de lo discutido en las reuniones. Aunque en los diarios apenas han realizado referencias a la importancia de los mismos. durante las distintas entrevistas y en las memorias sí han argumentado su gran utilidad para favorecer la reflexión.

Ha sido curioso el caso de la alumna D, la cual manifestando en un principio que era lo que menos le atraía del programa porque no se consideraba muy constantey no le gustaba escribir, una vez iniciado el programa lo

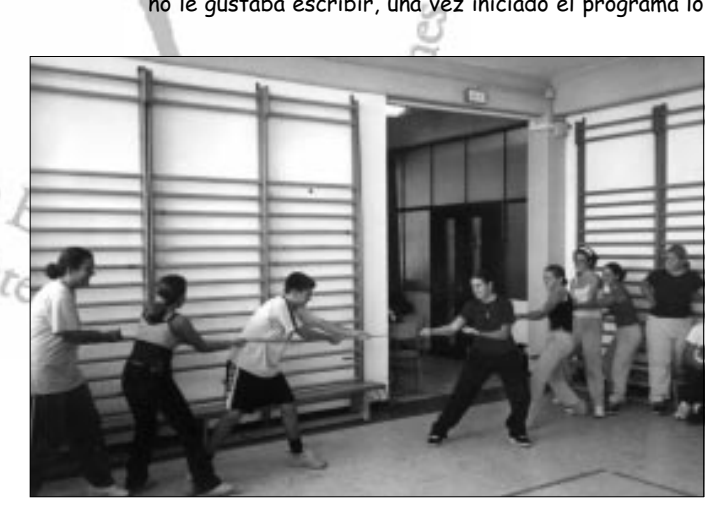

ha defendido y, además, ha elaborado unos diarios bastante detallados.

"Al principio no me gustó, no me encuentro a gusto y tengo la sensación de que todo lo que yo quisiera contar, en el diario siempre se me olvidan cosas, pero a medida que me obligaron a hacer el diario, me ayudaba por un lado a reflejar más o menos lo que habiamos hecho durante las reuniones y en las clases, (...) Por otro lado me ayudan a hacer otra reflexión; veníamos hablábamos y había una reflexión dentro de la reunión: pero yo cuando me voy sigo pensando y el diario refleja una reflexión más personal. (...) Si pudiera, hoy no lo evitaría. (Entrevista a la alumna D. 18-2-98)" (Toja, 2001, p.380)

Entendemos que la mejor defensa que respecto a su utilidad han podido hacer se encuentra en sus propios diarios, pues todos y cada uno de ellos reflejan un permanente diálogo de cada una de las alumnas consigo misma, casi a modo de un amigo imaginario. Además éstos en ningún caso son simples memorias descriptivas $y$, por el contrario, todos reflejan niveles de reflexión elevados en los que no sólo se enumera sino también se describe, se explica y se ofrecen alternativas. Veamos algunas citas que pueden 
ejemplificar lo que decimos pero que difícilmente muestran la evolución del pensamiento.

"Participar del calentamiento me acercó a los alumnos, al trabajar con grupos procuré ir pasando por todos y sacándoles lo que tienen dentro y no identifican como valor, sus ideas valen, también los apoyo a veces en el proceso de completarlo con propuestas e ideas. procuro darles siempre varias opciones, quizás dirijo demasiado para que salgan cosas y vean que esto tiene sentido." (Alumna A. Diario de Prácticas. 16-2-98)

"Elaboré una ficha para cada alumno donde recojo después de cada sesión: el grado de participación, motivación, esfuerzo interés, el nivel de aprendizaje, características de su personalidad, carencias importantes y qué comportamiento debo tomar yo ante él para ayudarle en su formación. Creo que, aunque en un princimás justa, es muy útil para conocer mejor a los alumnos, reflexionar sobre ellos, sus posibles problemas y sobre el tipo de actuación que debería tener el alumno." (Alumn B. Diario de Prácticas. 8-1-98)

"... esto confirma la necesaria contemplación en la práctica de aquella teoría aparentemente utópica del trato individualizado de los alumnos. Sólo tratando de conectar con su pensamiento, sus deseos, o sus manifestaciones ex ternas, es posible respetar las diferencias sin convertirlas en discriminación y sin generar, de paso, una relación fría y ausente." (Alumna C. Diario de Reuniones. 12-2-1998)

"¿Son tan importantes nuestros problemas como para obsesionarse con ellos? iestamos actuando como realmente nos habiamos preparado y conforme con nues tros ideales? ¿qué estamos haciendo?, ¿cuáles son mis tros ideales?, ¿qué estamos haciendo?, ¿cuáles son mis objetivos como profesora de Educación Física?, ¿son los rio de Reuniones. 16-4-977

En ellos ha quedado reflejado el aprendizaje experiencial de cada una de las alumnas y su maduración en el proceso reflexivo.

"Los futuros profesionales igual que aprenden a ac tuar, deberian aprender a reflexionar sobre la actuación Es decir, debería acostumbrarse a afrontar la actuación profesional de una manera reflexiva. Eso es lo que debe proporcionar las prácticas a los estudiantes: ayudarles a proporcionar las prácticas a los estudiantes: ayudarles a asentar su futuro desarrollo profesional en los parámetró reflexión-acción de contraste.)"(Zabalza, 1990: 33)

\section{Principales aportaciones del diario: Conclusiones de un encuentro}

Con el paso del tiempo hemos comprobado cómo los diarios respondieron a las expectativas que habíamos creado acerca de sus posibilidades para la formación.

Su utilidad para la recogida de información en la investigación-acción genera las siguientes posibilidades de actuación:

- Facilitar la descripción de los pensamientos, opiniones y concepciones teóricas.
"No hay un método bueno, lo que hay son alumnos." (Alumna B. Diario de Reuniones. 15-1-1997)

- Permite conocer las preocupaciones que los/as alumnos/as presentan frente a la práctica a lo largo de todo el proceso.

"¿Cómo mides el interés en la clase?. ¿Se puede sistematizar este aspecto? Tal vez con un diario de cada clase donde se anote el interés de cada alumno. Pero tiene un problema y son los alumnos grises, aquellos que pasan totalmente desapercibidos. (Alumna C. Diario de Reuniones. 10-4-98)

- Mostrar las estrategias de intervención realizadas por los/as alumnos/as y la justificación que hacen de las mismas.

"Con este grupo debo hacer modificaciones. Creo que debo ir en progresión tanto con el grupo como con el contenido. Creo que si empiezo con un componente más lúdico y poco a poco introduzco el elemento expresivo me funcionará más el grupo que si lo hago de repente." (Alumna D. Diario de Prácticas. 6-2-98)

- Explicitar los dilemas que se producen entre sus concepción teórica y su forma de proceder en la práctica, así como otro tipo de dilemas.

"Muy bien (tristemente, teniendo en cuenta el "método presionante" que empleé) los hábitos higiénicos! Estoy consiguiendo muchas cosas, aunque tengo que reconocer que ello me obliga a sacrificar algunas otras en las que creo firmemente (los 15' de la ducha - intentaré reducirlos, naturalmente). Llegué a la conclusión de que cualquier actuación es correcta (incluso si no se ajusta a un modelo que yo creía ideal y pedagógicamente correcto) si los objetivos se consiguen con un ajuste a lo que los alumnos piden." (Alumna C. Diario de Prácticas. 4-2-98)

- Reflejar cuáles son, día a día, los pensamientos respecto a la participación en este programa, frente a otros miembros del grupo, las técnicas utilizadas, los procesos seguidos, etc.

"Cambió en algo nuestra idea de E.F. desde que comenzamos las prácticas? Pienso que la ajusté a un alumnado real. Al adolescente de hoy. "(Alumna A. Diario de Reuniones. 2-3-98)

- Se convierte en el principal instrumento de triangulación. Por una parte, facilita el proceso de análisis inicialmente inductivo: la lectura de cada uno de los diarios dio paso al análisis del resto de los documentos; y por otra, marcó el camino a seguir en la discusión de los resultados y la triangulación final. El diario nos ha permitido realizar la triangulación de tiempos, de cada sujeto consigo mismo, de sujetos (contrastando distintos diarios) y de técnicas (comparando con otros instrumentos).

En los últimos años, con otros grupos que han seguido este programa hemos confirmado que el diario es un importante instrumento que facilita la reflexión sobre la práctica y mejora la futura intervención.

Siendo conscientes de que la elaboración del diario es un compromiso que en un primer momento puede parecer excesivo, vistos los resultados durante estos años no dudamos que su realización debe ser promovida en la asignatura de Practicum y en los programas de Investi- 


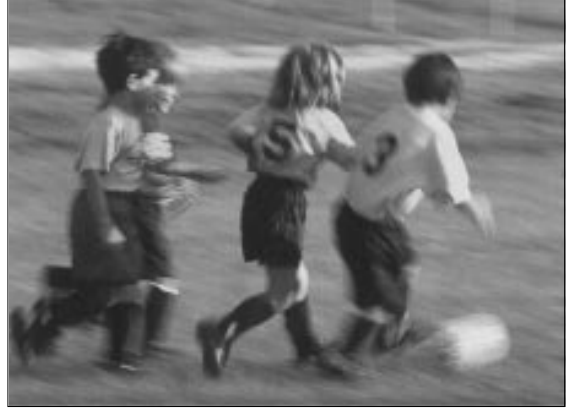

gación-Acción fomentarlo en todos los integrantes del grupo de investigación hasta convertirlo en un hábito cotidiano. Con ello estaremos sirviendo a un doble objetivo: la formación inicial y la formación permanente.

"El diario escolar se convierte en un valioso instrumento que posibilita el abandono de las acciones robotizadas y rutinarias en el aula como pauta primordial de conducta. Al mismo tiempo, permite la aparición de la acción reflexiva y la potenciación de la capacidad de los docentes como generadores del conocimiento profesional." (Torres Santomé,1986: 52)

\section{Bibliografía}

DEL VILLAR, F. (1994). El diario de los profesores de educación física. Un instrumento de investigación y formación docente. Revista Español de Educación Física y Deportes, vol. 1, $n^{\circ} 4$, pp. 20-23.

FRAILE, A. (1995). El maestro de educación física y su desarrollo profesional. Salamanca: Amarú.

GARCÍA RUSO, H. (1992). La formación del profesorado de Educación Física: una propuesta de curriculum basada en la reflexión en la acción. Universidad de Santiago de Compostela. Tesis doctoral no publicada.

GONZÁLEZ SANMAMED, M. (1994). Aprender a enseñar: Mitos y realidades. A Coruña: Servicio de Publicacións da Universidade da Coruña.

MARCELO C (1994) Formación del profesorado para el cambio educativo. Barcelona: PPU.

MEDINA, J. (1995). Influencia de un entrenamiento docente basado en el trabajo en grupo durante la for mación inicial del profesorado de Educación Física. Un estudio preliminar. Universidad de Granada. Tesis doctoral no publicada.

RAMOS, L. A. (1999). La evolución del pensamiento docente de los profesores de Educación Física a través de un programa de supervisión orientado a la reflexión en la acción y sobre la acción. Universidad de Extremadura. Tesis no publicada.

RODRÍGUEZ LÓPEZ, J. M. a (1995). Formación de profesores y prácticas de enseñanza. Un estudio de caso. Huelva: Servicio de Publicaciones Universidad de Huelva.
ROMERO CEREZO, C. (1995). Incidencia de un programa de formación inicial del maestro especialista en educación física en los niveles de reflexión y toma de decisiones sobre la práctica docente. Universidad de Granada. Tesis doctoral no publicada.

SCHÖN, D. (1983). The reflective practitioner. How professionals think in action. New York: Basic Book, Inc., Publishers.

SCHÖN, D. (1987). Educating the reflective practitioner. San Francisco: Josey Bass Publishers.

TOJA, B. , CARREIRO, F. Y GONZÁLEZ VALEIRO M. A. (1998). La Investigación-Acción dentro de un programa de Practicum en el Instituto Nacional de Educación Física de Galicia. En Ma N. González de la Hoz, C. Lobato y MaP. Ruíz Ojeda (comps), Desarrollo Profesional y Practicum en la Universidad, pp.287-295.

TOJA, B., GONZALEZ VALEIRO, M.A. FERNÁNDEZ VILLARINO, M.A.,GARCÍA TURNES, S., GESTEIRO, M.C., JUEGA, B., MOINELO, M.A. Y PILLADO, N. (1999a): "La investigaciónacción: Una alternativa par los programas de formación inicial en el ámbito de la licenciatura en Ciencias de la actividad física y el deporte: Justificación y preparación del proyecto". Revista de Educación Física, $n^{\circ} 75$, págs. $5-9$

TOJA, B., GONZALEZ VALEIRO, M.A. FERNÁNDEZ VILLARINO M.A GARCÍA TURNES S. GESTEIRO, M.C., JUEGA, B., MOINELO, M.A. Y PILLADO, N. (1999b): "La investigación-acción: Una alternativa para los programas de formación inicial en el ámbito de la licenciatura en Ciencias de la actividad física y el deporte: Intervención docente y análisis". Revista de Educación Física, $n^{\circ} 76$, págs. 15-19

TOJA, B. (2001). Estudio de un programa de formación en preservicio en Educación Física: La Investigación-Acción como estrategia de formación e investigación. Universidad de A Coruña. Tesis doctoral no publicada.

TORRES SANTOMÉ, J. (1986). El diario escolar. Cuadernos de pedagogía, n 142, pp. 52-55.

YINGER CH.M y CLARK R. (1981). Reflective journal writting: theory and practice. Paper, $n^{\circ} 50$. East Lansing, Michigan: Michigan State University, The Institute for Research on Teaching.

YINGER CH. M. y CLARK R. (1985). Using personal documents to study teacher thinking. Paper $n^{\circ} 84$. East Lansing, Michigan: Michigan State University, The Institute for Research on Teaching

YINGER CH. M. y CLARK R. (1988). El uso de documentos personales en el estudio del pensamiento del profesor. En L. Villar Angulo (dir.) Conocimiento, creencias y teorías de los profesores. Implicaciones para el curriculum y la formación del profesorado. Alcoy: Marfil, pp. 175-196.

ZABALZA, M. A. (1990) Teoría de las prácticas. La formación práctica de los profesores. Actas del II Simposium sobre prácticas escolares. Poio, pp. 25-37.

ZABALZA, M. A. (1991). Los diarios de clase. Documento para estudiar cualitativamente los dilemas prácticos de los profesores. Barcelona: PPU. 